\title{
CASO -2018: Bacteremia primaria por Pasteurella multocida en un paciente con hepatopatía crónica y cáncer de pulmón
}

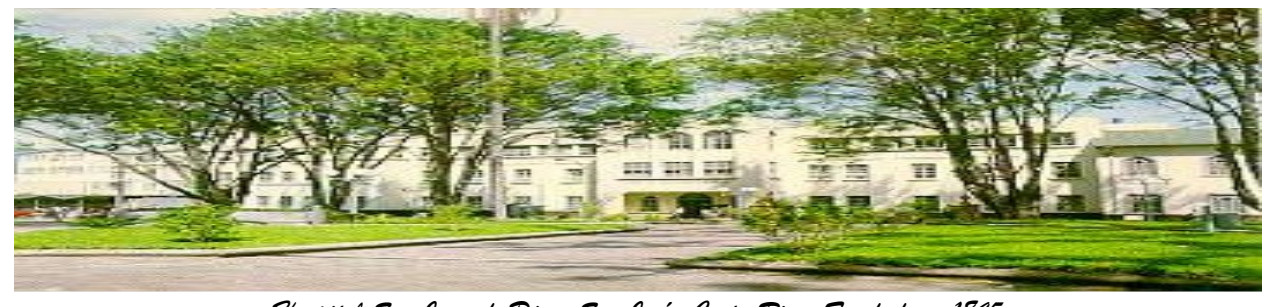

Frospital San quan de Dias. San Gosé, Costa Rica. Fundado en 1845

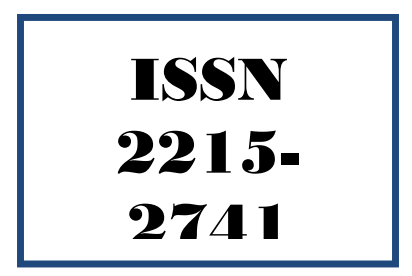

Recibido:

$08 / 02 / 2018$

Aceptado:

$15 / 03 / 2018$

Edith Barrantes Valverde ${ }^{1}$

Ana Isabel Herrera Bogantes ${ }^{2}$

Manuel Antonio Villalobos Zúñiga ${ }^{3}$

${ }^{1}$ Microbióloga Especialista en Bacteriología. Laboratorio de Bacteriología del Hospital San Juan de Dios.

${ }^{2}$ MQC. Laboratorio de Bacteriología del Hospital San Juan de Dios.

${ }^{3}$ Médico Especialista en Infectología y Medicina Interna. Hospital San Juan de Dios.

manuel701@gmail.com

\section{RESUMEN}

Se presenta a continuación el caso de un paciente masculino de 47 años, con antecedente de hepatopatía crónica clasificada como Child Pugh B secundaria a infección por el virus de Hepatitis $\mathrm{B}$, diabetes mellitus 2 e hipertensión arterial, quien consulta al servicio de emergencias por 5 meses de evolución de disnea en reposo, tos productiva y hemoptisis; habiéndose descartado tuberculosis se documenta con neumonía intersticial y bacteremia por Pasteurella multocida. En este mismo ingreso se diagnostica con cáncer de pulmón indiferenciado.

\section{PALABRAS CLAVE}

Bacteremia. Hepatopatía crónica. Pasteurella multocida

\section{ABSTRACT}

This is the case of a 47-year-old male patient with a history of chronic liver disease classified as Child B secondary to hepatitis B, diabetes mellitus 2 and hypertension, who went to the emergency room due to 5 months of dyspnea at rest, productive cough and hemoptysis; lung infection due to tuberculosis was ruled out, while interstitial pneumonia and bacteremia are documented by Pasteurella multocida. The diagnosis of lung cancer was also made in the current hospitalization.

\section{KEY WORDS}

Bacteremia. Chronic hepatopathy. Pasteurella multocida 


\section{INTRODUCCIÓN}

El género Pasteurella, nombrado así en honor a Louis Pasteur, posee varias especies la mayoría de importancia en medicina veterinaria. ${ }^{(1,2)}$ Afecta un amplio grupo de animales: ovejas, ratones, ganado, pájaros, perros y gatos ${ }^{(2,3,4)}$; sin embargo, Pasteurella multocida es la especie más común como causa de infecciones oportunistas en humanos, así como también ocasionando infecciones en mordeduras de perro y gato fundamentalmente. Autores como Wilson y Ho consideran que debido a la alta prevalencia de esta bacteria como parte de la microbiota en animales domésticos y salvajes y a la cada vez más frecuente interacción con humanos, la transmisión zoonotica de P. multocida es un riesgo para la infección. ${ }^{(2)}$ Hay evidencia de que actualmente la pasteurelosis está aumentando a nivel global. ${ }^{(5)}$

P. multocida existe como comensal en tracto gastrointestinal, mucosa nasal, orofaríngea ${ }^{(3)} \mathrm{y}$ tracto respiratorio de animales domésticos e incluso humanos. ${ }^{(3,6)}$ La fuente de infección más frecuente es por contaminación de heridas con saliva animal o por secreciones aerosolizadas ${ }^{(3)}$ que permiten la colonización del árbol traqueobronquial de los propietarios de mascotas.

Este agente causa varios procesos infecciosos, los más comunes en piel y tejidos blandos, frecuentemente luego de rasguños o mordeduras por gatos o perros, quienes son portadores entre $70 \%-90 \%$ y $20 \%-50 \%$ respectivamente. ${ }^{(1)} \mathrm{El}$ tracto respiratorio es el segundo sitio más común de infección. ${ }^{(7)}$ La mayoría de cuadros de neumonía se presentan en adultos mayores con enfermedad pulmonar crónica, pero pueden verse también infecciones de vía aérea superior y abscesos. ${ }^{(8,9)} \mathrm{La}$ enfermedad invasiva se ha reportado en pacientes de alto riesgo que presentan enfermedades malignas, trasplantes de órganos y cirrosis. ${ }^{(2,10)}$ Es una enfermedad poco común en pacientes inmunocompetentes. ${ }^{(3,1)}$

P. multocida es un bacilo gram negativo de 1-2 $\mu \mathrm{m}$ de largo, que varía de formas bacilares ovales cortas a cocobacilares. Se presentan únicas, en pares ó en cadenas y es no móvil. Crece bien en agar sangre, no crece en agar Mac ConKey, es oxidasa positiva y fermentador de carbohidratos; además posee varios factores de virulencia como su capacidad de sobrevivir en el medio ambiente, su mecanismo de adquisición de hierro, su cápsula antifagocítica y adhesinas. ${ }^{(2,5)}$ Enzimas extracelulares como la hialuronidasa, neuraminidasa y proteasas facilitan la colonización y diseminación. El lipopolisacárido le confiere sero-resistencia. Algunas cepas muy virulentas tienen toxina dermonecrótica con capacidad de regular la respuesta inmune.

\section{CASO CLÍNICO}

Masculino de 47 años vecino de Cariari en Pococí de Limón. Portador de diabetes mellitus tipo 2 , hipertensión arterial además de hepatopatía crónica con estadío clínico Child Pugh B, secundaria a infección por virus de la hepatitis $\mathrm{B}$, con hipertensión portal, várices esofágicas e hiperesplenismo. El tratamiento crónico de este paciente es tenofovir $300 \mathrm{mg}$ vo por día, omeprazol 20 $\mathrm{mg}$ vo por día, propanolol $40 \mathrm{mg}$ vo por día, ácido fólico $1 \mathrm{mg}$ vo por día, lactulosa $30 \mathrm{ml}$ tres veces al día, espironolactona $100 \mathrm{mg}$ por día e insulina glargina (lantus $\left.{ }^{\circledR}\right) 40$ Uds por día. En lo relativo a su hepatopatía crónica no ha tenido episodios de sangrado variceal y se encuentra estable con su última carga viral por hepatitis $\mathrm{B}<$ 10 copias $/ \mathrm{mL}$; sin embargo, su diabetes no tiene un adecuado control y aunque no tiene daño a órgano blanco evidente, tiene hemoglobinas glicosiladas subóptimas, es decir $\mathrm{HbAlC}>7 \%$. ${ }^{(12)} \mathrm{El}$ paciente no tiene antecedentes epidemiológicos relevantes excepto que vive en una región tropical húmeda como la provincia de Limón.

Este paciente consulta por 5 meses de evolución de disnea en reposo, tos productiva y hemoptisis. Se descartó Tuberculosis de forma ambulatoria previamente, y consulta en esta ocasión por exacerbación de sus síntomas respiratorios, fiebre y síntomas constitucionales. Ingresa febril, taquicárdico, sudoroso, normotenso, con adecuada saturación de oxígeno, sin datos clínicos de encefalopatía hepática, con auscultación cardiopulmonar sin hallazgos patológicos, abdomen blando sin ascitis. La radiografía de tórax muestra un infiltrado intersticial.

Los exámenes de laboratorio a su ingreso en el servicio de emergencia fueron los siguientes: $\mathrm{He}-$ 
moglobina: 13,4 g/dl, Leucocitos 7900 / uL, Bandas $11 \%$, Plaquetas 56,000 / uL, Creatinina: $0,68 \mathrm{mg} / \mathrm{dl}$, BUN: $21 \mathrm{mg} / \mathrm{dl}$, Glicemia: 187 mg/dl, ELISA por HIV negativo, HbA1C: 8,2 \%, TP: 23 seg, INR: 2.03, TPT: 38 seg, VES: 21 mm3, PCR:11,1 mg/dl. Los hemocultivos son reportados positivos en ambas botellas tomadas de ambos miembros superiores: 13 y 12 horas respectivamente, por Pasteurella multocida. Con respecto a estudios de gabinete, se realiza Broncoscopía, con cultivo de aspirado bronquial positivo por Streptococcus parasanguis y PCR negativa por tuberculosis. El resultado de la biopsia mostró Cáncer de pulmón indiferenciado. Las demás pruebas de laboratorio obtenidas a lo largo de su hospitalización no difieren mayormente de las iniciales anotadas previamente, cabe señalar aquí, que los biomarcadores mejoraron en su totalidad con la terapia instaurada.

Ante la bacteremia que presenta el paciente, se interroga específicamente acerca del contacto con mascotas (gatos o perros), lo cual niega enfáticamente. Sin embargo en su casa si hay un perro, pero él insiste en que está fuera de la casa y él no mantiene ningún contacto directo con él.

El manejo clínico de este caso se realizó desde un inicio de forma empírica con Cefotaxime a dosis usuales de $2 \mathrm{~g}$ intravenoso cada 8 horas por 10 días; por lo tanto, su desenlace clínico fue adecuado en esta hospitalización, y el paciente pudo egresar con control posterior en oncología y en gastroenterología.

\section{MICROBIOLOGÍA}

En el laboratorio se recibieron dos frascos de hemocultivo de este paciente que resultaron positivos a las 12 y 13 horas de incubación en equipo automatizado (Bact/Alert, bioMérieux, Marcy l'Étoile, Francia). Al realizar la tinción de Gram se observaron cocobacilos gramnegativos. Se realizaron subcultivos de cada frasco en $\mathrm{Me}$ dio de Columbia con 5\% de sangre de carnero y Agar chocolate (bioMérieux, Marcy l'Étoile,Francia). Se incubaron en atmósfera de $5 \%$ de $\mathrm{CO}_{2}$ a $37{ }^{\circ} \mathrm{C}$ por 24 horas, obteniéndose crecimiento de colonias lisas pequeñas, no hemolíticas de color grisáceo correspondientes a un cocobacilo gramnegativo, catalasa positivo, oxidasa positivo. La identificación en tarjeta $\mathrm{GN}$ Vi- tek 2 Compact ( bioMérieux , Marcy l'Étoile,Francia ) resultó Pasteurella multocida con $95 \%$ de probabilidad en ambos aislamientos.

La prueba de sensibilidad a los antibióticos en tarjeta AST-279 ( bioMérieux ) se reporta sensible para Betalactámicos, Cefalosporinas de tercera generación, Carbapenémicos, Ciprofloxacina, y Trimetoprima- Sulfametoxazol, sensibilidad intermedia para Gentamicina y resistente para Amikacina. No se realizó sensibilidad a macrólidos.

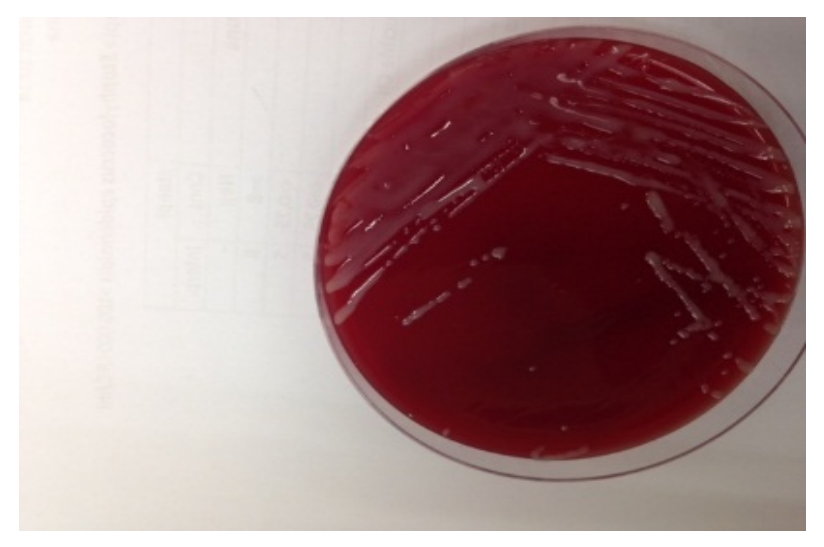

Figura 1. Cultivo de Pasteurella multocida en placas de agar Columbia a las 48 horas de incubación.

Fuente: Laboratorio de Bacteriología. HSJD.

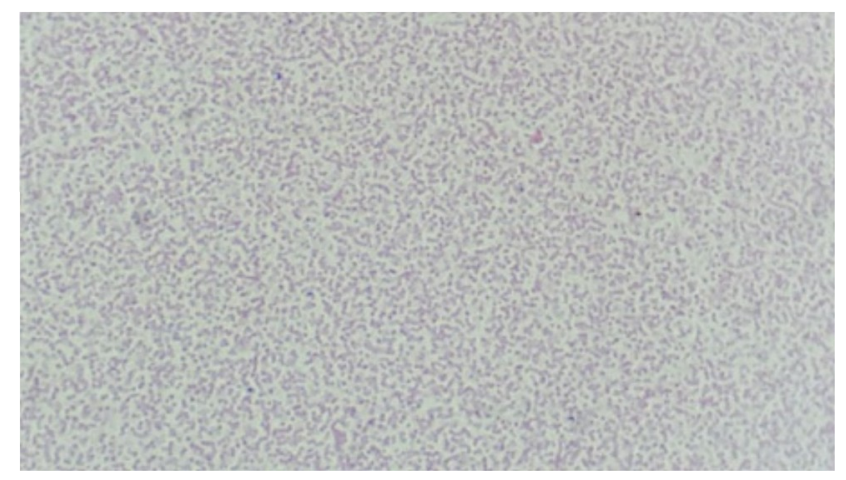

Figura 2 . Tinción de Gram mostrando Bacilos Gram Negativos (Pasteurella multocida), realizada a partir del cultivo.

Fuente: Laboratorio de Bacteriología. HSJD. 


\section{DISCUSIÓN Y ANÁLISIS DEL CASO}

El diagnóstico de infecciones por Pasteurella spp. requiere de la obtención de información detallada del paciente en la historia clínica sobretodo en cuanto a edad, estado inmunológico, enfermedades concomitantes, ocupación e historia de contacto con animales, particularmente mordeduras o contacto cercano. ${ }^{(7)}$

P. multocida es un patógeno potencial que debe ser considerado como causa de bacteremia en pacientes inmunocomprometidos y en aquellos que reciben quimioterapia que induce a neutropenia. ${ }^{(3,12)}$ La disfunción hepática (cirrosis de cualquier etiología, hepatitis y tumores infiltrantes) se ha reportado como un factor de riesgo asociado a bacteremia debido a la alteración del sistema reticuloendotelial. ${ }^{(6,10)}$ De hecho, los factores de riesgo con mayor asociación a la enfermedad invasiva por $P$. multocida son la enfermedad hepática, el tabaco y el alcohol, incluso sobre otras formas de inmunosupresión. ${ }^{(13)}$

En pacientes con neumonía y bacteremia por $P$. multocida, factores como el fumado que altera la función del sistema mucociliar y del sistema inmune a nivel pulmonar, y la enfermedad pulmonar estructural, son aspectos que propician la inoculación directa y la colonización de las vías respiratorias por esta bacteria. ${ }^{(3,14)}$ La presentación radiológica más común es la neumonía intersticial como el caso que nos ocupa, a la cual también se le conoce como neumonía atípica. ${ }^{(15)}$ Otra forma descrita de compromiso respiratorio es el absceso pulmonar, existiendo casos tanto en inmunocompetentes como inmunocomprometidos, así como también infecciones de la vía aérea superior ${ }^{(8,16,17)}$

Se ha reportado el aislamiento de este microorganismo de muestras de heridas, esputo, lavado bronquioalveolar, líquido ascítico, articular, pleural y cefalorraquídeo. ${ }^{(18)}$ La meningitis se asocia tanto a infección por continuidad a partir de una sinusitis como al antecedente de trauma o neurocirugía previa, siendo ésta una de las formas más graves de la infección. ${ }^{(19,20)}$ La infección en torrente circulatorio no es comúnmente reportada y ocurre por diseminación a partir de un foco infeccioso, ya sea por herida por mordedura ó en algunos casos sin que exista un foco aparente como se ha reportado en la literatura en pacientes con infección diseminada. ${ }^{(7)}$ En algunos casos se reporta una historia de contacto con animales ${ }^{(10,21)}$ pero en la mayoría se desconoce la fuente de la bacteremia, e incluso hay reportes de bacteremia recurrente por este germen. ${ }^{(6,18,19)}$

La bacteremia en este paciente está probablemente asociada a múltiples factores de riesgo: hepatopatía crónica, hipertensión portal e hiperesplenismo, diabetes mellitus tipo 2 sin control óptimo y el cáncer de pulmón indiferenciado de reciente diagnóstico. Aunque el paciente refiere poco contacto directo con animales, en el ambiente domiciliar si menciona la presencia de un perro, por lo que existe la probabilidad de colonización de mucosas por aerosoles y la posterior multiplicación bacteriana, permitiendo generar la bacteremia manifiesta en los cultivos realizados.

Al realizar el diagnóstico de laboratorio, se debe recordar la similitud de la morfología de especies de Pasteurella con las bacterias del grupo HACEK (Haemophilus, Actinobacillus, Cardiobacterium, Eikenella, Kingella) y de Capnocytophaga spp ${ }^{(21)}$, además de otras como Moraxella, Neisseria y Acinetobacter. El crecimiento de la bacteria en Agar Columbia es lento, por lo se debe de esperar las 48 horas de incubación para realizar las pruebas de identificación y sensibilidad a los antibióticos. Las pruebas de oxidasa y catalasa son positivas, el crecimiento en agar sangre es de colonias grisáceas no hemolíticas. No es necesario su aislamiento en Agar chocolate debido a que su crecimiento no depende del factor X, a diferencia de Haemophilus spp.

En relación a la sensibilidad a los antibióticos, el patógeno presentó un patrón similar al reportado en la literatura ${ }^{(3)}$, exceptuando la sensibilidad a macrólidos, para la cual se advierte precaución en su uso especialmente como monoterapia, debido a la presencia de aislamientos resistentes a este grupo de antibióticos, para el que en nuestro caso no se realizó la prueba. Cabe señalar que la sensibilidad en las infecciones primarias es similar a aquellas asociadas a mordeduras de gatos o perros ${ }^{(22),}$ por lo tanto el presente reporte tiene validez en ambos escenarios y sirve de referencia para elegir tratamientos ambulatorios o parenterales, siendo que el aislamiento de este germen es infrecuente. 
Debido a que la infección por $P$. multocida se comporta como un oportunista, es importante enfatizar las medidas de prevención en pacientes de riesgo. En este sentido, se recomienda extremar las medidas de higiene en el manejo de las mascotas de riesgo, es decir en gatos, perros, aves y conejos; optimizando el lavado de manos, asegurando la salud del animal con el cuidado veterinario requerido, evitando el contacto con animales con diarrea, y en general limitando el contacto con secresiones orales de las mascotas - lamidos - sobretodo en heridas o piel no íntegra. En algunos casos es recomendable utilizar equipo de protección personal como guantes y mascarillas para la manipulación de los animales. ${ }^{(23)}$ Las vacunas anti $P$. multocida, pretenden disminuir la infección en animales ya que esta causa importantes pérdidas económicas a este nivel; sin embargo, esto podría eventualmente disminuir la infección en humanos ya que se disminuiría su reservorio más importante. ${ }^{(24)}$

En Costa Rica no se conoce la prevalencia de $P$. multocida, sin embargo es muy importante obtener una historia bien detallada del contacto con animales en pacientes inmunocomprometidos, de la tercera edad, con enfermedades malignas, tratamiento inmunosupresor, trastornos hepáticos, tumores infiltrantes y patologías pulmonares en quienes se presente un cuadro clínico infeccioso sospechoso de infección por esta bacteria, que se reporta como causante de una mortalidad del $7 \%$ al $30 \%$ por bacteremia, sin foco de ingreso conocido.

Según la revisión de la literatura correspondiente el presente es el primer el reporte de un caso clínico de bacteremia primaria por $P$. multocida en Costa Rica. En la literatura médica del pais figura un reporte de casos de absceso cerebral en niños donde se menciona este agente, y en otro donde aparece como colonizador de ventiladores y aires acondicionados en hospitales nacionales. ${ }^{(25,26)}$ Esto confirma en cierta medida lo poco común de este aislamiento microbiológico y apoya la importancia del presente reporte de caso.

\section{CONCLUSIONES}

Se ha reportado el primer caso en Costa Rica de bacteremia primaria por P. multocida en un paciente inmunocomprometido, con la intención de ampliar el conocimiento clínico de esta bacteria y sus alcances en la medicina humana. Es así como este microorganismo puede comportarse como un agente oportunista invasivo en individuos con patologías crónicas e inmunosupresión y no sólo como la bacteria asociada comúnmente a las mordeduras por animales.

\section{BIBLIOGRAFÍA}

1. Ferreira J, Treger K, Busey K. Pneumonia and disseminated bacteremia with Pasteurella multocida in the immune competent host: A case report and a review of the literature. Respir Med Case Reports [Internet]. 2015;15:54-6. Available from: http://dx.doi.org/10.1016/j.rmcr.2015.04.005

2. Wilson BA, Ho M. Pasteurella multocida: from Zoonosis to Cellular Microbiology. Clin Microbiol Rev. 2013;26(3):631-55.

3. Casey AC, Greenspoon JS, Lagasse LD, Al CET. Pasteurella multocida Bacteremia in a Patient With Ovarian Cancer and Chemotherapy-Induced Neutropenia. Inf Dis Obstet Gynecol. 1996;209(C):205-9.

4. Hendrie J. Pasteurella multocida bacteremia in humans a clinical report. Can Fam Physician. 1974;20(4):79-81.

5. Wilson BA, Ho M. Pasteurella multocida: From Zoonosis to cellular microbiology. Clin Microbiol Rev. 2013;26(3):631-55.

6. Miranda I, Amaya MAJ V. Revista Española de Cirugía Ortopédica y Traumatología Infección aguda de prótesis total de rodilla tras mordedura y ara nazo de gato : caso clínico y revisión de la bibliografía. Rev Esp Cir Ortop Traumatol [Internet]. 2013;57(4):300-5. Available from: http://dx.doi.org/10.1016/j.recot.2013.04.006

7. Kofteridis DP, Christofaki M, Mantadakis E, et al. Bacteremic community-acquired 
pneumonia due to Pasteurella multocida. Int $\mathrm{J}$ Infect Dis. 2009;13(3):81-3.

8. Schmulewitz L, Chandesris MO, Mainardi JL, et al. Invasive Pasteurella multocida sinusitis in a renal transplant patient. Transpl Infect Dis. 2008;10(3):206-8.

9. Hazouard E, Ferrandiére M, Brunereau $\mathrm{L}$ et al. Fulminant Pasteurella multocida lung abscess in a chronic alcohol abuser: diagnosis using intracavital lavage. Intensive Care Med. 2000 Jul $3 ; 26(9 \quad$ UIhttp://icmjournal.esicm.org/journal/134/0/9/2609 1404/0/):1404-5.

10. Tseng H-K, Su S-C, Liu C-P LC-M. Pasturella multocida bacteremia due to non-bite animal exposure in cirrhotic patients: report of two cases. J Microbiol Immunol Infect. 2001;34:293-6.

11. Giordano A, Dincman T, Clyburn BE, Steed LL, et al. Clinical Features and Outcomes of Pasteurella multocida Infection. Med. 2015;94(36):1-7.

12. Care D, Suppl SS. 6. Glycemic Targets: Standards of Medical Care in Diabetes-2018. Diabetes Care [Internet]. 2018;41(Supplement 1):S55-64. Available from: http://care.diabetesjournals.org/lookup/doi/10.2337/dc18-S006

13. Nollet V, Souply L, Rosolen B, et al. Risk factors for invasive pasteurellosis: a retrospective case study. Eur J Clin Microbiol Infect Dis [Internet]. 2016;35(12):1975-81. Available from: $\quad$ http://dx.doi.org/10.1007/s10096-0162749-y

14. Harper M, Boyce JD, Adler B. Pasteurella multocida pathogenesis : 125 years after Pasteur. Fed Eur Microb Soc Microbiolol. 2006;265(1):1-10.

15. Schilichthaar H, Rohrer T, Schuster G et al. Interstitial pneumoniae and sepsis due to a Pasteurella multocida infection. Dtsch Med Wochenschr. 1995;120(46):1582-6.

16. Machiels P, Haxhe JP, Trigaux JP, et al. Chronic lung abscess due to Pasteurella multocida. Thorax [Internet]. 1995;50(9):1017-8.
Available from: http://www.pubmedcentral.nih.gov/articlerender.fcgi?artid=1021325\&tool=pmcentrez\&rendertype $=$ abstract

17. Goussard P, Gie RP, Steyn F, et al. Pasteurella multocida lung and liver abscess in an immune-competent child. Pediatr Pulmonol. 2006;41(3):275-8.

18. Narsana N, Farhat F. Septic shock due to Pasteurella multocida bacteremia : a case report. J Med Case Rep [Internet]. 2015;9-11. Available from: http://dx.doi.org/10.1186/s13256-015-0643-3

19. Zarlasht F, Khan M. A Case of Recurrent Pasteurella Bacteremia in an Immunocompetent Patient with No Animal Bite. Am J Case Rep [Internet]. 2018;19:95-8. Available from: https://www.amjcaserep.com/abstract/index/idAr $\underline{\mathrm{t} / 907251}$

20. López C, Sanchez-Rubio P, Betrán A, et al. Pasteurella multocida bacterial meningitis caused by contact with pigs. Brazilian J Microbiol. 2013;44(2):473-4.

21. Soloaga R, Carrión N, Pidone J, et al. Meningitis bacteriémica por Pasteurella multocida. Rev Argent Microbiol. 2008;40(4):208-10.

22. Ludwig C, de Jong A, Moyaert $\mathrm{H}$, et al. Antimicrobial susceptibility monitoring of dermatological bacterial pathogens isolated from diseased dogs and cats across Europe (ComPath results). J Appl Microbiol. 2016;121(5):1254-67.

23. Christenson ES, Ahmed HM, Durand CM. Pasteurella multocida infection in solid organ transplantation. Lancet Infect Dis [Internet]. 2015;15(2):235-40. Available from: http://dx.doi.org/10.1016/S1473-3099(14)70895-3

24. Ahmad TA, Tawfik DM, Sheweita SA, et al. Development of immunization trials against Pasteurella multocida. Trials Vaccinol [Internet]. 2016;5(8):53-60. Available from: http://dx.doi.org/10.1016/j.vaccine.2013.11.068

25. Sáenz-Llorens XJ, Umaña $M$ a, Odio $\mathrm{CM}$, et al. Brain abscess in infants and children. Pediatr Infect Dis J [Internet]. 1989;8(7). Avail- 
able

from:

http://journals.lww.com/pidj/Fulltext/1989/07000

/Brain_abscess_in_infants and children_9.aspx

26. Gamboa MM, Rodríguez E RM. Bacterias de importancia clínica en respiradores y aires acondicionados de hospitales de San José, Costa Rica. Rev Biomed. 2003;14(3):14351.

\section{CONFLICTO DE INTERÉS Y/O AGRADECIMIENTOS}

Los autores declaran que no existió ningún conflicto de interés en el presente reporte. 